\title{
Functional anatomy: dynamic states in basal ganglia circuits
}

\author{
Marianela Garcia-Munoz, Luis Carrillo-Reid and Gordon W. Arbuthnott*
}

Brain Mechanisms for Behaviour Unit, Okinawa Institute for Science and Technology, Onna, Okinawa, Japan

Edited by:

Jose L. Lanciego, University of

Navarra, Spain

Reviewed by:

Noritaka Ichinohe, Hirosaki University, Japan

Antonio Pereira, Federal University of Rio Grande do Norte, Brazil

Manuel Alegre, University of Navarra,

Spain

\section{${ }^{*}$ Correspondence:}

Gordon W. Arbuthnott, Brain

Mechanisms for Behaviour Unit,

Okinawa Institute for Science and

Technology, 1919-1 Tancha, Onna-son,

Onna, Okinawa, Japan.

e-mail: gordon@oist.jp
The most appealing models of how the basal ganglia function propose distributed patterns of cortical activity selectively interacting with striatal networks to yield the execution of contextdependent movements. If movement is encoded by patterns of activity then these may be disrupted by influences at once more subtle and more devastating than the increase or decrease of neuronal firing that dominate the usual models of the circuit. In the absence of dopamine the compositional capabilities of cell assemblies in the network could be disrupted by the generation of dominant synchronous activity that engages most of the system. Experimental evidence about Parkinson's disease suggests that dopamine loss produces abnormal patterns of activity in different nuclei. For example, increased oscillatory activity arises in the GPe, GPi, and STN and is reflected as increased cortical beta frequency coherence disrupting the ability to produce motor sequences. When the idea of deep brain stimulation was proposed - it was supported by the information that lesions of the subthalamus reversed the effects of damage to the dopamine input to the system. However, it seems increasingly unlikely that the stimulation acts by silencing the nucleus as was at first proposed. Perhaps the increased cortical beta activity caused by the lack of dopamine could have disabled the patterning of network activity. Stimulation of the subthalamic nucleus disrupts the on-going cortical rhythms. Subsequently asynchronous firing is reinstated and striatal cell assemblies and the whole basal ganglia circuit engage in a more normal pattern of activity. We will review the different variables involved in the generation of sequential activity patterns, integrate our data on deep brain stimulation and network population dynamics, and thus provide a novel interpretation of functional aspects of basal ganglia circuitry.

Keywords: neuronal networks, striatum, cell assemblies, compositional properties, deep brain stimulation, calcium imaging, synaptic plasticity, intrinsic neuronal properties

\section{INTRODUCTION}

Global changes in the activity of specific basal ganglia structures generate devastating pathologies (e.g., Parkinson's disease; Huntington chorea). Interpreting the overall behavior of the group of structures called basal ganglia is fundamental for the understanding of motor disorders.

The most predominant model of basal ganglia function is based on the existence of two striatal pathways with antagonistic effects (Albin et al., 1989) the activation of the direct pathway facilitates the execution of movements, and the activation of the indirect pathway inhibits motion (Takakusaki et al., 2004a,b). So it was proposed that movement alterations in Parkinson's disease are caused by the over activation of the indirect pathway, whereas hyperkinetic disorders reflect the pathologically increased activity in the direct pathway. Initially it seemed that this simplification had some merit, since lesions in the indirect pathway helped Parkinson's disease symptoms (Bergman et al., 1990).

\section{A MORE REALISTIC MODEL IS NEEDED}

However, the reality of basal ganglia encoding is likely to be more complex. Not only is such simple coding unlikely to be versatile enough to encompass motor skills but the basal ganglia are also involved in learning such skills (Graybiel, 2000; Yin et al., 2009). We imagine that groups of neurons in several of the nuclei of the basal ganglia could work together in dynamic networks to accomplish these tasks. Recurrent and alternating activity of specific groups of cells could interact to form the substrate that gives rise to the processes associated with learning. Neuronal pools with these characteristics have been called cell assemblies (Hebb, 1949). In order to store and perform complex behaviors, cell assemblies must have diverse capabilities, such as the ability to synchronize activity and to compose sequences among different neuronal microcircuits (Hebb, 1949; Carrillo-Reid et al., 2009a). Compositionality is a central issue to cognitive processes; it refers to the ability to generate complex procedures from basic patterns represented by the coordinated activity of specialized neurons organized in hierarchies (Bienenstock and Geman, 1995; Hammer, 2003).

\section{A BETTER SCHEME?}

We would like to present the hypothesis that the compositional capabilities observed in neuronal microcircuits can explain normal and pathological behaviors of the basal ganglia. From this point of view, diverse functions can be represented by the continuous reorganization of specialized cell assemblies to accommodate new habit learning and automaticity in learned motor or cognitive tasks. It has been reported for instance that different striatal areas change their predominant activity as learning progresses (Lehericy et al., 2005). Indeed the final memory may be laid down outside the basal ganglia in cerebellum (Doyon et al., 2009). The next challenge is to develop novel analytical tools and experimental techniques 
to define the dynamic functional anatomy of the basal ganglia. The study of how anatomy is related to the function of a system requires the understanding of different aspects that shape neural networks. The three main points that can describe the organization of specialized groups of neurons are: (1) the intrinsic properties of the neurons, (2) the synaptic dynamics, and (3) the compositional properties of several interconnected structures.

\section{INTRINSIC PROPERTIES OF NEURONS}

The most abundant cell type in the striatum is the medium spiny neuron which is characterized by a hyperpolarized resting membrane potential and low input resistance (Kita et al., 1984) as well as several types of potassium conductance that shape their firing patterns (Nisenbaum and Wilson, 1995). Membrane depolarization and spiking yield calcium influx, calcium influx activates small and large conductance calcium-activated potassium channels (Bargas et al., 1999) and therefore limits cell firing.

\section{PLATEAU POTENTIALS}

Medium spiny neurons display periodically changing firing patterns, referred to as "up and down" states, according to variations in their intrinsic membrane properties as well as excitatory drive from the cortex and thalamus (Wilson and Kawaguchi, 1996). The up state persists as long as sufficient excitatory drive is present to maintain depolarization (Wilson, 1993; Vergara et al., 2003).

Active conductances that give rise to plateau potentials play an important role in the generation of oscillations in several areas. A plateau potential is a stable membrane potential kept above the resting membrane potential. When a plateau potential is generated the cells can produce action potentials in the absence of sustained synaptic excitation (Grillner et al., 1981; Guertin and Hounsgaard, 1998a; Vergara et al., 2003). In this way, a transient depolarization of sufficient amplitude and duration can initiate a plateau potential. The plateau can last several seconds before ending spontaneously or it can be terminated by an inhibitory synaptic input. Moreover, plateau potentials are regulated by different neuromodulators for instance in spinal motor neurons (Guertin and Hounsgaard, 1998b).

A strong synchronous input is not always necessary to induce bursts of action potentials in several cells at the same time. It is often enough to have similar temporal sequences to induce prolonged activity even if the temporal sequences come from a different source (Carrillo-Reid et al., 2008).

Network dynamics that depend on plateau potentials have been observed in different structures of the basal ganglia (Vergara et al., 2003; Ibanez-Sandoval et al., 2007; Carrillo-Reid et al., 2008, 2009a,b). For example, it has been shown that plateau potentials underlie up and down state transitions in medium spiny projection neurons from the striatum (Vergara et al., 2003; Carrillo-Reid et al., 2008). In vivo experiments have revealed that the bursting activity of these neurons reflects specific motor patterns (Vautrelle et al., 2009). At present here are no ways to evaluate the presence and significance of plateau potentials in intact organisms, including humans (Kiehn and Eken, 1998).

\section{SYNAPTIC DYNAMICS}

Excitability changes at the presynaptic level that modify neurotransmitter release occur at all levels of the central nervous system with important consequences in synaptic communication. Activation of presynaptic receptors and modulation of secretion is a well-recognized event (de Jong and Verhage, 2009). In the basal ganglia presynaptic influences on dopamine release were reported more than thirty years ago (reviewed by Glowinski et al., 1979) followed some years later by presynaptic receptor mediated changes in axonal terminal excitability (Garcia-Munoz et al., 1991a,b). Since the first descriptions of corticostriatal synaptic plasticity were made on the corticostriatal terminals (Garcia-Munoz et al., 1992) the majority of presynaptic studies have examined synaptic plasticity and transmission at excitatory glutamatergic synapses on striatal output neurons (Ding et al., 2008; Kreitzer and Malenka, 2008). Short-term synaptic plasticity is also involved in the stabilization and reconfiguration of motor circuits and in the initiation, maintenance, and modulation of programs related to movement (Nadim and Manor, 2000). In the production of striatal short-term and long-term synaptic changes (potentiation and depression) the participation of glutamate and dopamine receptors has been demonstrated (Calabresi et al., 1992; Wickens et al., 1996; Kerr and Wickens, 2001; Tecuapetla et al., 2007).

In the model proposed by Hebb (1949) coincidental sustained firing is necessary to increase synaptic efficacy, a necessary characteristic to allow the transmission between short-term and long-term memory. With this in mind, the synchronous activation of specific microcircuits could modulate network synaptic efficacy promoting the long-term storage of mental representations (Tallon-Baudry et al., 2001).

Short-term synaptic plasticity can be used to dynamically select among different motor patterns. It can be used not only to select different patterns of movement, but also different motor programs (e.g., swimming, walking). Synaptic plasticity is dependent on activity and is regulated by neuromodulators, sensory experience or a combination of both (Nadim and Manor, 2000).

The induction of striatal short-term and long-term synaptic changes is related to habit formation but also to pathological states (Ingham et al., 1998; Costa et al., 2006; Day et al., 2006; Kreitzer and Malenka, 2008; Yin et al., 2009). In like manner the understanding of the synaptic dynamics between different structures of the basal ganglia could be a fundamental key to define the functional anatomy underlying motor related behaviors.

\section{COMPOSITIONAL PROPERTIES AND BASAL GANGLIA FUNCTION}

Procedural memories and habits can be represented by basic motor actions (Graybiel, 1998; Grillner, 2006). Basic modules can be combined to generate a broad repertoire of procedures used to perform specific tasks. The knowledge of the compositional rules that guide the formation of habits is fundamental to understanding the dynamic states underlying basal ganglia functions (CarrilloReid et al., 2008, 2009a). Nevertheless, newer techniques must be developed to investigate experimentally the functional connectivity between specialized groups of cells and behavioral events.

Repetitive practice can create procedural memories allowing the consolidation of specific patterns of motor activity. A memory system based on modular activity has the advantage that any module can retrieve the whole memory (Abeles et al., 2004; Carrillo-Reid et al., 2009a). Activation of recurrent sequences (i.e., neuronal patterns) has been related to working memory (Lewis et al., 2005a,b). Sustained frontal cortical activity represents working 
memory processes (Kessler et al., 2005; Pollok et al., 2006) and the sustained corticostriatal activity has been proposed to participate in the planning and control of movements (Beiser et al., 1997). Distributed patterns of cortical activity selectively interacting with striatal networks may underlie the execution of context-dependent movements (Stern et al., 1998; Costa et al., 2006; Yin et al., 2009).

\section{A REDUCED SYSTEM}

In vitro preparations allow the easy access to neuronal assemblies making it possible to study the transfer of patterns of activity. By plating and growing cortical and striatal neurons in different compartments of a multielectrode array we have found that the activity patterns in the cortical compartment are followed by striatal neurons (Figure 1).

These preliminary experiments suggest that the behavior of cortical and striatal networks is present in greatly simplified systems but the results need to be expanded both in the details of the analysis of the activity and in applying similar methods to other basal ganglia networks. For instance these cultures have no dopamine input but it could be added.

There are many theories of brain function that depend on cell assemblies with the ability to reproduce sequential patterns of activity that can be "remembered" when a small sample of the network is ignited (Bienenstock and Geman, 1995; Abeles, 2003; Hammer, 2003; Abeles et al., 2004; Grillner, 2006). There are many fewer experiments showing that real nerve cells do produce such "compositional" networks (Ikegaya et al., 2004; Carrillo-Reid et al., 2008, 2009a).

In electrical recordings from cortical cells the subthreshold membrane potential state transitions are correlated with the slow rhythm of the electroencephalogram. Clear transitions seen in the anesthetized animal are replaced by oscillations in the firing of groups of neurons in "microzones" or "ensembles" that are time locked to specific movements (Jaeger et al., 1995).

\section{CALCIUM IMAGING AS A METHOD TO EXAMINE ENSEMBLES}

The use of calcium imaging techniques in corticostriatal slices has revealed that active up states of medium spiny neurons are accompanied by calcium influx (Vergara et al., 2003; Carrillo-Reid et al.,
2008, 2009a). When NMDA is applied in vitro to striatal slices, it induces recruitment of active neurons and generates dispersed patterns of activity in the network. Neurons belonging to the network alternate their activity to generate spatiotemporal patterns of synchronization (Carrillo-Reid et al., 2008).

Research on how such striatal neuronal assemblies are modified by neurotransmitters and neuromodulators has just begun. So far we know that modifications in GABA and acetylcholine transmission have important consequences (Figure 2). GABA receptor blockade locks the network in a recurrent pattern of activity. Activation of cholinergic muscarinic receptors induces a change in network dynamics by recruiting neurons into correlated firing without increasing the number of active neurons. The presence of acetylcholine seems to allow the formation of cell assemblies with different cycles of activity. These results are important inasmuch as they suggest that the cortical and thalamic glutamate inputs are not the only modulators of striatal network activity. GABA, dopamine and acetylcholine participate in typical sequential activation of cell assemblies, the key element in the generation of motor programs. Although we have looked at these in striatal neurons we expect similar properties to be present throughout the basal ganglia circuits.

It is proposed that activation of motor commands induces recruitment of active neurons and generates stable patterns of activity in striatal neurons that are preserved in vitro. Neurons belonging to specific network states alternate their activity to generate spatiotemporal patterns of synchronization (Carrillo-Reid et al., 2008, 2009a; Jaidar et al., 2010). Thus GABA, dopamine and acetylcholine participate in typical sequential activation of cell assemblies, the key element in the generation of motor programs (Figure 2).

\section{CLINICAL IMPLICATIONS}

The importance of these network properties for understanding basal ganglia function may not lie only in their intrinsic biological interest. In recent experiments on the underlying mechanism of the therapeutic effects of deep brain stimulation we were forced to conclude that the effect might be a consequence of action at the

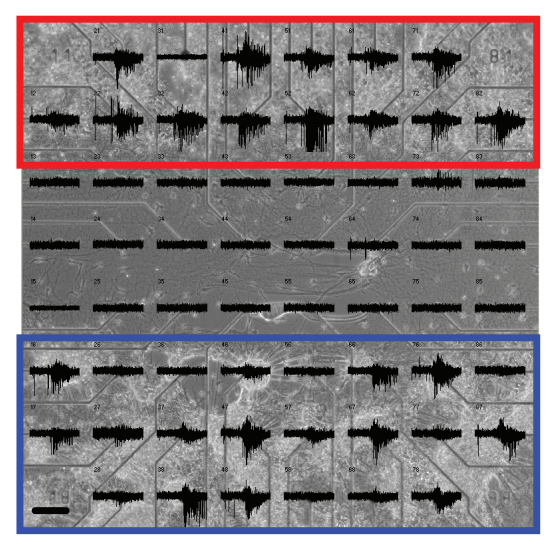

FIGURE 1 | Cortical networks can transfer information to striatal networks. Left: microelectrode array (MEA) recordings of a compartmentalized culture of cortical cells (red) and striatal cells (blue). Note that cortical activity can trigger the activity of striatal networks. Scale bar $100 \mu \mathrm{V} / 600 \mathrm{~ms}$. Right: raster plot

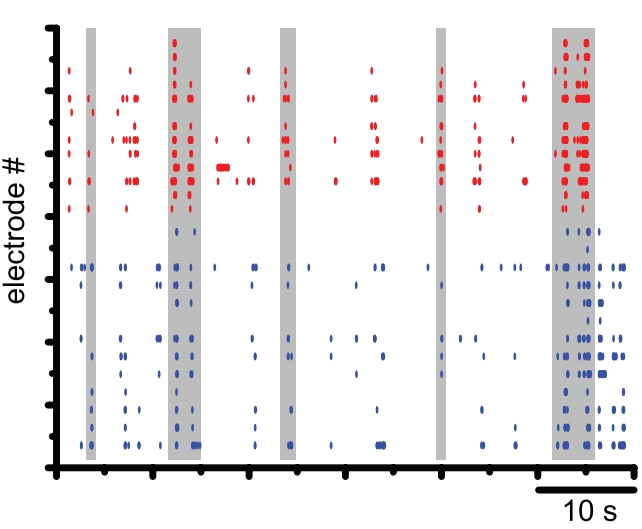

representing the activity recorded by the cortical electrodes (red) and the striatal electrodes (blue). The overall activity of the network seems to be defined by synchronous firing of the cortical neurons. Gray stripes indicate the entrainment of firing in the striatal compartment in time with the cortical activity. 

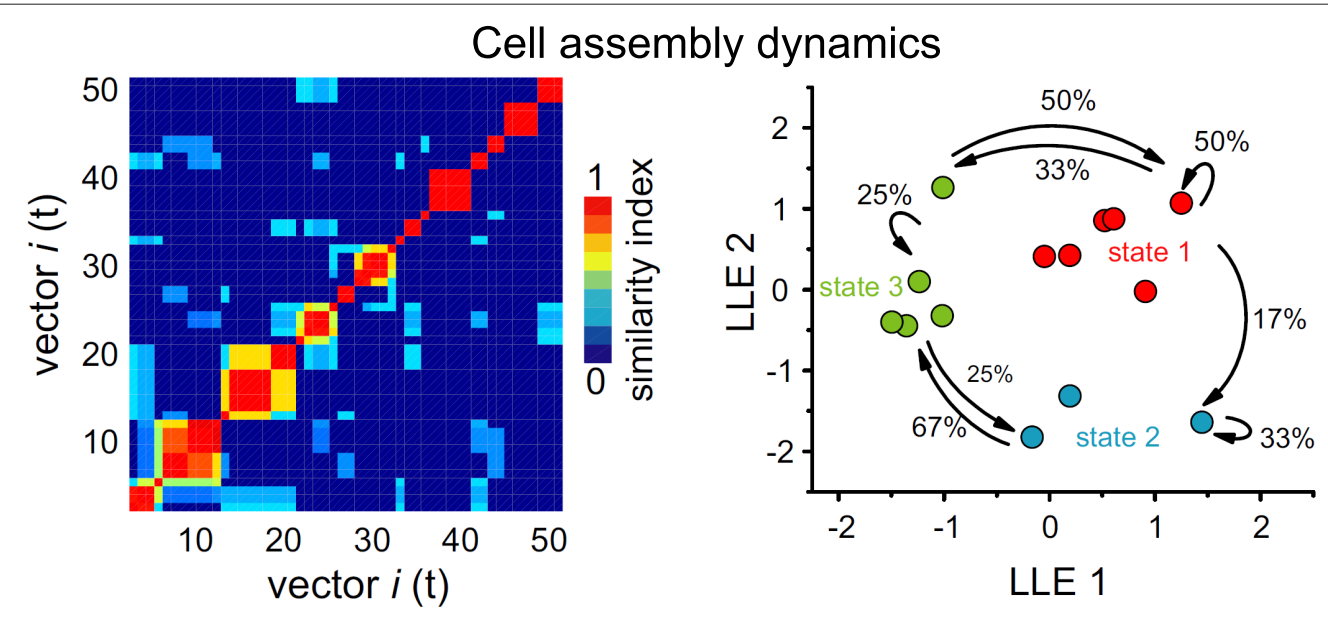

Compositional properties of cell assemblies
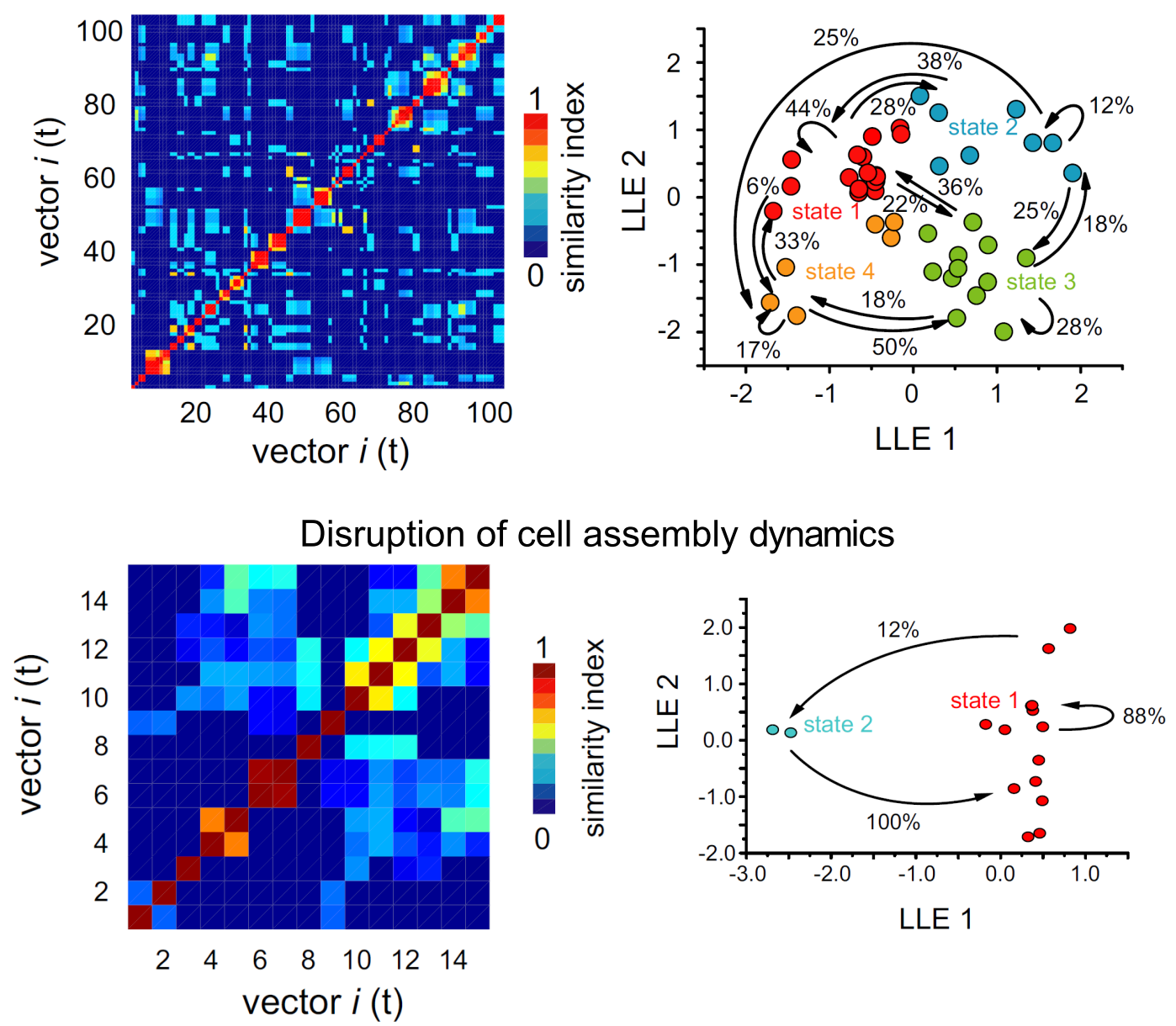

FIGURE 2 | Cell assembly dynamics in cortico-striatal slices. Left: maps showing the similarity index of the vectors representing network dynamics as a function of time. The vectors are generated from the firing of individual cells over time, each cell vector is compared with every other to generate the similarity index plot. Patterned structures indicate groups of cells firing together at different times. Right: two dimensional projection using locally linear embedding (LLE) of the vectors defining the states of the network. Arrows indicate transitions between different states. Top: NMDA receptor activation produced synchronous, recurrent and alternating activity in striatal neuronal pools. All these properties are characteristics of cell assemblies. Modified from Carrillo-Reid et al. (2009a). Middle: activation of the cholinergic system endows cell assemblies with compositional capabilities allowing the formation of complex sequences of activity from elemental patterns. Modified from Carrillo-Reid et al. (2009a). Bottom: blockade of GABA receptors engages the striatal network in a dominant state. Note the drastic readjustment of cell assemblies from diverse cycles of activity with compositional properties to a fixed pattern that reduced the cell assembly diversity. Modified from Carrillo-Reid et al. (2008). 
cortex - and not in the subthalamic nucleus, where the electrodes are aimed. One obvious effect of the stimulation on cortical activity is to suppress the beta waves in the ECoG that are induced by blocking dopamine receptors.

The drug effect is small compared to the result of lesions of the dopamine system (Mallet et al., 2008a,b), but the akinesia produced is profound and that also is alleviated by stimulation that reduces the beta frequency power in the ECoG (Dejean et al., 2009; Figure 3).

In contemporaneous but not directly related experiments Deisseroth's group (Gradinaru et al., 2009) used optogenetic techniques to influence the cells of the nucleus. They showed that inactivating much of the subthalamic nucleus, or activating the cells there, produced no effect on the symptoms caused by lesion of dopamine cells in the mouse. However, they could influence the behavioral effects of the lesion by applying light to activate the cells of layer $\mathrm{V}$ of the cortex. These are the same cells that it had been previously shown to be antidromically activated by deep brain stimulation in the rat subthalamic nucleus (Li et al., 2007; Lehmkuhle et al., 2009).

\section{CORTICAL INVOLVEMENT IN DEEP BRAIN STIMULATION}

Thus, disrupting the rhythmic activity in the cortex that gives rise to the beta rhythm in the ECoG is capable of relieving the symptoms of basal ganglia dopamine depletion. This suggests that the beta activity is somehow changing the basal ganglia dynamic in a way that disrupts its function (Arbuthnott et al., 2009). One interpretation of these results is that the patterned activity that we have imaged in striatal cultures and in slices of the striatum, is a vital part of the normal activity that underlies voluntary movement. Its disruption by cortical beta dominance blocks the compositional network activity, and so incapacitates the animal - or the patient.

Recovery after deep brain stimulation, or after magnetic stimulation of the cortex (Fregni and Pascual-Leone, 2007) may be a consequence of the disruption of this cortical rhythm and as a result removing its domination of the network activity in the striatum. Return the striatum to normal patterned activity and the animal can move and behave again; the Parkinsonian patient can move again. Of course the disruption of cortical activity itself has consequences that may entail "cognitive side effects" of stimulation. If thoughts and memories depend on compositional network activity then it is not a surprise that stimulation also interrupts those in cortex at least to some extent.

\section{CONCLUSIONS}

We have tried to introduce new concepts indicating that basal ganglia structures are capable of forming neuronal networks that can demonstrate compositional properties.

The concept of a basic stereotypic microcircuit formed by several neurons with common connections and dynamic operations can invigorate basal ganglia research. Storage and retrieval of learned

\section{REFERENCES}

Abeles, M. (2003). "Synfire chains," in The Handbook of Brain Theory and Neural Networks, ed. M. A. Arbib (Cambridge, MA: The MIT Press), 1143-1146.
Abeles, M., Hayon, G., and Lehmann, D. (2004). Modeling compositionality by dynamic binding of synfire chains. $J$. Comput. Neurosci. 17, 179-201.

Albin, R. L., Young, A. B., and Penney, J. B. (1989). The functional anatomy

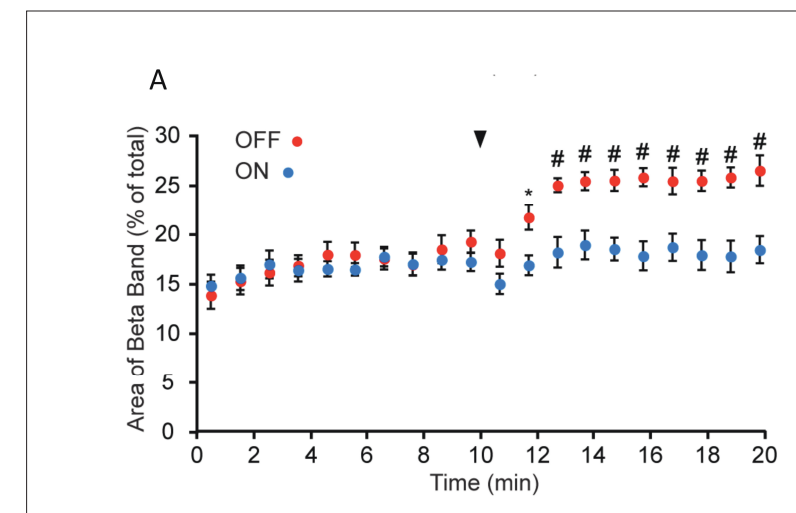

B

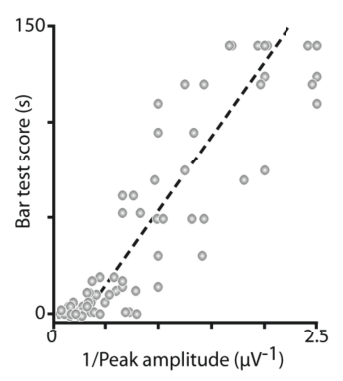

FIGURE 3 | Cortical effects and the recovery from akinesia. In (A) the average percentage power in the beta band of the EEG is plotted for all nine rats in the study. Each rat is recorded in two conditions. The red symbols are from a day when the rats were treated with DI and D2 blockers after 10 min in the recording chamber and for 10 min thereafter. The blue symbols represent 2 days later when the animals were again in the same recording chamber but with the stimulation $\mathrm{ON}(120 \mathrm{~Hz}$ and less than $80 \%$ of the threshold for movement). The beta power increase caused by the drugs is blocked. In (B) we show the inverse relationship of the effect to the stimulus strength. The threshold for a cortical evoked potential is just below $2 \mu \mathrm{A}$ ( 0.5 on the scale). The animals come off the bar very quickly when the stimulus is above threshold and the recovery is directly related to the inverse of the stimulus power (more effective akinesia prevention is proportional to higher current $\left.\left(r^{2}=0.84\right)\right)$.

patterns of movement could take place in the basal ganglia by the dynamic reorganization of cell assemblies. To make predictions of future events and generate appropriate behavior, our cortex and basal ganglia could store sequences of patterns and retrieve suitable sequences by their similarity with previously successful patterns.

The generation of predictive knowledge of the properties of the world around is vital to our ability to act upon it and within it. Not surprising then that the perceptual processes often thought of as vital for such knowledge of the body in the world should be visible within the basal ganglia. Informed, or disrupted by cortical influences, maintained by the action of different neurotransmitters, our normal lives depend on the compositional properties of diverse cell assemblies.

of basal ganglia disorders. Trends Neurosci. 12, 366-375.

Arbuthnott, G., Dejean, C., and Hyland, B. (2009). "Antidromic cortical activity as the source of therapeutic actions of deep brain stimulation?" in Cortico-Subcortical Dynamics in Parkinson's Disease, ed. K. Y. Tseng (New York: Humana Press), 393-403.

Bargas, J., Ayala, G. X., Vilchis, C., Pineda, J. C., and Galarraga, E. (1999). 
Ca2+-activated outward currents in neostriatal neurons. Neuroscience 88 479-488.

Beiser, D. G., Hua, S. E., and Houk, J. C. (1997). Network models of the basal ganglia. Curr. Opin. Neurobiol. 7, 185-190.

Bergman, H., Wichmann, T., and DeLong, M. R. (1990). Reversal of experimental Parkinsonism by lesions of the subthalamic nucleus. Science 240, 1436-1438.

Bienenstock, E., and Geman, S. (1995). "Compositionality," in The Handbook of Brain Theory and Neural Networks, ed. M.A. Arbib (Cambridge, MA: The MIT Press), 223-226.

Calabresi, P., Maj, R., Mercuri, N. B., and Bernardi, G. (1992). Coactivation of D 1 and D 2 dopamine receptors is required for long-term synaptic depression in the striatum. Neurosci. Lett. 142, 95-99.

Carrillo-Reid, L., Tecuapetla, F., IbanezSandoval, O., Hernandez-Cruz, A., Galarraga, E., and Bargas, J. (2009a). Activation of the cholinergic system endows compositional properties to striatal cell assemblies. J. Neurophysiol. 101, 737-749.

Carrillo-Reid, L., Tecuapetla, F., Vautrelle, N., Hernandez, A., Vergara, R., Galarraga, E., and Bargas, J. (2009b). Muscarinic enhancement of persistent sodium current synchronizes striatal medium spiny neurons. J. Neurophysiol. 102, 682-690.

Carrillo-Reid, L., Tecuapetla, F., Tapia, D., Hernandez-Cruz, A., Galarraga, E., Drucker-Colin, R., and Bargas, J. (2008). Encoding network states by striatal cell assemblies. J. Neurophysiol. 99, 1435-1450.

Costa, R. M., Lin, S. C., Sotnikova, T. D., Cyr, M., Gainetdinov, R. R., Caron, M. G., and Nicolelis, M. A. (2006). Rapid alterations in corticostriatal ensemble coordination during acute dopaminedependent motor dysfunction. Neuron 52, 359-369.

Day, M., Wang, Z., Ding, J., An, X., Ingham, C. A., Shering, A. F., Wokosin, D., Ilijic, E., Sun, Z., Sampson, A. R., Mugnaini, E., Deutch, A. Y., Sesack, S. R., Arbuthnott, G. W., and Surmeier, D. J. (2006). Selective elimination of glutamatergic synapses on striatopallidal neurons in Parkinson disease models. Nat. Neurosci. 9, 251-259.

de Jong, A. P. H., and Verhage, M. (2009). Presynaptic signal transduction pathways that modulate synaptic transmission. Curr. Opin. Neurobiol. 19, 245-253.

Dejean, C., Hyland, B., and Arbuthnott, G. (2009). Cortical effects of subthalamic stimulation correlate with behavioral recovery from dopamine antagonist induced akinesia. Cereb. Cortex 19 1055-1063.

Ding, J., Peterson, J. D., and Surmeier, D. J. (2008). Corticostriatal and thalamostriatal synapses have distinctive properties. J. Neurosci. 28, 6483-6492.

Doyon, J., Bellec, P., Amsel, R., Penhune, V., Monchi, O., Carrier, J., LehĖricy, S., and Benali, H. (2009). Contributions of the basal ganglia and functionally related brain structures to motor learning. Behav. Brain Res. 199, 61-75.

Fregni, F., and Pascual-Leone, A. (2007). Technology insight: noninvasive brain stimulation in neurology-perspectives on the therapeutic potential of rTMS and tDCS. Nat. Clin. Pract. Neurol. 3, 383-393.

Garcia-Munoz, M., Young, S. J., and Groves, P. M. (1991a). Terminal excitability of the corticostriatal pathway. Il. Regulation by glutamate receptor stimulation. Brain Res. Bull. 551, 207-215.

Garcia-Munoz, M., Young, S. J., and Groves, P. M. (1991b). Terminal excitability of the corticostriatal pathway. I. Regulation by dopamine receptor stimulation. Brain Res. Bull. 551, 195-206.

Garcia-Munoz, M., Young, S. J., and Groves, P.M. (1992). Presynaptic longterm changes in excitability of the corticostriatal pathway. Neuroreport 3, 357-360.

Glowinski, J., Cheramy, A., and Giorguieff, M. F. (1979). "In-vivo and in-vitro release of dopamine," in The Neurobiology of Dopamine, eds A. S. Horn, J. Korf, and B. H. C. Westerink (London: Academic Press), 199-216.

Gradinaru, V., Mogri, M., Thompson, K. R., Henderson, J. M., and Deisseroth, K. (2009). Optical deconstruction of Parkinsonian neural circuitry. Science 324, 354-359.

Graybiel, A. M. (1998). The basal ganglia and chunking of action repertoires. Neurobiol. Learn. Mem. 70, 119-136.

Graybiel, A. M. (2000). The basal ganglia. Curr. Biol. 10, R509-R511.

Grillner, S. (2006). Biological pattern generation: the cellular and computational logic of networks in motion. Neuron 52, 751-766.

Grillner, S., McClellan, A., Sigvardt, K., Wallen, P., and Wilen, M. (1981). Activation of NMDA-receptors elicits "fictive locomotion" in lamprey spinal cord in vitro. Acta Physiol. Scand. 113, 549-551.

Guertin, P. A., and Hounsgaard, J. (1998a). NMDA-Induced intrinsic voltage oscillations depend on L-type calcium channels in spinal motoneurons of adult turtles. J. Neurophysiol. 80, 3380-3382.
Guertin, P.A., and Hounsgaard, J. (1998b). Chemical and electrical stimulation induce rhythmic motor activity in an in vitro preparation of the spinal cord from adult turtles. Neurosci. Lett. 245, 5-8.

Hammer, B. (2003). "Compositionality in neural systems," in The Handbook of Brain Theory and Neural Networks, 2nd Edn., ed. M.A.Arbib (Cambridge, MA: The MIT Press), 244-248.

Hebb, D. O. (1949). The Organization of Behavior; A Neuropsychological Theory. New York: Wiley.

Ibanez-Sandoval, O., Carrillo-Reid, L., Galarraga, E., Tapia, D., Mendoza, E., Gomora, J. C., Aceves, J., and Bargas, J. (2007). Bursting in substantia nigra pars reticulata neurons in vitro: possible relevance for Parkinson disease. J. Neurophysiol. 98, 2311-2323.

Ikegaya, Y., Aaron, G., Cossart, R., Aronov, D., Lampl, I., Ferster, D., and Yuste, R. (2004). Synfire chains and cortical songs: temporal modules of cortical activity. Science 304, 559-564.

Ingham, C. A., Hood, S. H., Taggart, P., and Arbuthnott, G.W. (1998). Plasticity of synapses in the rat neostriatum after unilateral lesion of the nigrostriatal dopaminergic pathway. J. Neurosci. 18, 4732-4743.

Jaeger, D., Gilman, S., and Aldridge, J. W. (1995). Neuronal activity in the striatum and pallidum of primates related to the execution of externally cued reaching movements. Brain Res. 694, 111-127.

Jaidar, O., Carrillo-Reid, L., Hernandez, A., Drucker-Colin, R., Bargas, J. and Hernandez-Cruz, A. (2010). Dynamics of the Parkinsonian striatal microcircuit: entrainment into a dominant network state. J. Neurosci. 30, 11326-11336.

Kerr, J. N., and Wickens, J. R. (2001) Dopamine D-1/D-5 receptor activation is required for long-term potentiation in the rat neostriatum in vitro. J. Neurophysiol. 85, 117-124.

Kessler, K., Schmitz, F., Gross, J., Hommel, B., Shapiro, K., and Schnitzler, A (2005). Cortical mechanisms of attention in time: neural correlates of the Lag-1-sparing phenomenon. Eur. J. Neurosci. 21, 2563-2574.

Kiehn, O., and Eken, T. (1998). Functional role of plateau potentials in vertebrate motor neurons. Curr. Opin. Neurobiol. 8, 746-752.

Kita, T., Kita, H., and Kitai, S. T. (1984). Passive electrical membrane properties of rat neostriatal neurons in an in vitro slice preparation. Brain Res. 300, 129-139.

Kreitzer, A. C., and Malenka, R. C. (2008) Striatal plasticity and basal ganglia circuit function. Neuron 60, 543-554.
Lehericy, S., Benali, H., Van de Moortele, P. F., Pelegrini-Issac, M., Waechter, T., Ugurbil, K., and Doyon, J. (2005). Distinct basal ganglia territories are engaged in early and advanced motor sequence learning. Proc. Natl. Acad. Sci. U.S.A. 102, 12566-12571.

Lehmkuhle, M. J., Bhangoo, S. S., and Kipke, D. R. (2009). The electrocorticogram signal can be modulated with deep brain stimulation of the subthalamic nucleus in the hemiparkinsonian rat. J. Neurophysiol. 102, 1811-1820.

Lewis, D. A., Hashimoto, T., and Volk, D. W. (2005a). Cortical inhibitory neurons and schizophrenia. Nat. Rev. Neurosci. 6, 312-324.

Lewis, S. J. G., Slabosz, A., Robbins, T. W., Barker, R.A., and Owen,A.M. (2005b). Dopaminergic basis for deficits in working memory but not attentional set-shifting in Parkinson's disease. Neuropsychologia 43, 823-832.

Li, S., Arbuthnott, G. W., Jutrasi, M. J., Goldberg, J. A., and Jaeger, D. (2007). Resonant antidromic cortical circuit activation as a consequence of highfrequency subthalamic deep-brain stimulation. J. Neurophysiol. 98, 3525-3537.

Mallet, N., Pogosyan, A., Marton, L. F., Bolam, J. P., Brown, P., and Magill, P. J. (2008a). Parkinsonian beta oscillations in the external globus pallidus and their relationship with subthalamic nucleus activity. J. Neurosci. 28, 14245-14258.

Mallet, N., Pogosyan, A., Sharott, A., Csicsvari, J., Bolam, J. P., Brown, P., and Magill, P. J. (2008b). Disrupted dopamine transmission and the emergence of exaggerated beta oscillations in subthalamic nucleus and cerebral cortex. J. Neurosci. 28, 4795-4806.

Nadim, F., and Manor, Y. (2000). The role of short-term synaptic dynamics in motor control. Curr. Opin. Neurobiol. 10, 683-690.

Nisenbaum, E. S., and Wilson, C. J. (1995). Potassium currents responsible for inward and outward rectification in rat neostriatal spiny projection neurons. J. Neurosci. 15, 4449-4463.

Pollok, B., Gross, J., and Schnitzler, A. (2006). How the brain controls repetitive finger movements. J. Physiol. Paris 99, 8-13.

Stern, E. A., Jaeger, D., and Wilson, C. J. (1998). Membrane potential synchrony of simultaneously recorded striatal spiny neurons in vivo. Nature 394, 475-478.

Takakusaki, K., Saitoh, K., Harada, H., and Kashiwayanagi, M. (2004a). Role of basal ganglia-brainstem pathways in the control of motor behaviors. Neurosci. Res. 50, 137-151. 
Takakusaki, K., Oohinata-Sugimoto, J., Saitoh, K., and Habaguchi, T. (2004b). Role of basal ganglia-brainstem systems in the control of postural muscle tone and locomotion. Prog. Brain Res. 143, 231-237.

Tallon-Baudry, C., Bertrand, O., and Fischer, C. (2001). Oscillatory synchrony between human extrastriate areas during visual short-term memory maintenance. J. Neurosci. 21, RC177.

Tecuapetla, F., Carrillo-Reid, L., Bargas, J., and Galarraga, E. (2007). Dopaminergic modulation of shortterm synaptic plasticity at striatal inhibitory synapses. Proc. Natl. Acad. Sci. U.S.A. 104, 10258-10263.

Vautrelle, N., Carrillo-Reid, L., and Bargas, J. (2009). "Diversity of up- state voltage transitions during different network states," in CorticoSubcortical Dynamics in Parkinson Disease, ed. K. Y. Tseng (New York: Humana/Springer), 73-86.

Vergara, R., Rick, C., Hernandez-Lopez, S., Laville, J.A., Guzman, J. N., Galarraga, E., Surmeier, D. J., and Bargas, J. (2003). Spontaneous voltage oscillations in striatal projection neurons in a rat corticostriatal slice. J. Physiol. 553, 169-182.

Wickens, J. R., Begg, A. J., and Arbuthnott, G. W. (1996). Dopamine reverses the depression of rat corticostriatal synapses which normally follows high-frequency stimulation of cortex in vitro. Neuroscience 70, 1-6.
Wilson, C. J. (1993). The generation of natural firing patterns in neostriatal neurons. Prog. Brain Res. 99, 277-297.

Wilson, C. J., and Kawaguchi, Y. (1996). The origins of two-state spontaneous membrane potential fluctuations of neostriatal spiny neurons. J. Neurosci. 16, 2397-2410.

Yin, H. H., Mulcare, S. P., Hilario, M. R. F., Clouse, E., Holloway, T., Davis, M. I., Hansson, A. C., Lovinger, D. M., and Costa, R. M. (2009). Dynamic reorganization of striatal circuits during the acquisition and consolidation of a skill. Nat. Neurosci. 12, 333-341.

Conflict of Interest Statement: The authors declare that the research was conducted in the absence of any commercial or financial relationships that could be construed as a potential conflict of interest.

Received: 06 August 2010; accepted: 19 October 2010; published online: 23 November 2010.

Citation: Garcia-Munoz M, Carrillo-Reid $L$ and Arbuthnott GW (2010) Functional anatomy: dynamic states in basal ganglia circuits. Front. Neuroanat. 4:144. doi: 10.3389/fnana.2010.00144

Copyright (c) 2010 Garcia-Munoz, CarrilloReid and Arbuthnott. This is an open-access article subject to an exclusive license agreement between the authors and the Frontiers Research Foundation, which permits unrestricted use, distribution, and reproduction in any medium, provided the original authors and source are credited. 